\title{
Relación existente entre la temperatura del aceite del motor, régimen de giro y aceleraciones efectivas RMS, al utilizar gasolina y una mezcla entre gasolina- etanol
}

\section{Existing ratio between engine oil temperature, rotation rate and effective RMS acceleration, when using gasoline and a gasoline-ethanol mixture}

Mg. Andrés Castillo Reyes

Phd. Marcos Gutiérrez

Mario Cando

Bryan Calisto.

Universidad Internacional del Ecuador, Ecuador

Autor para correspondencia, acastillos@uide.edu.ec, marcosgutierrez@tablet-school.com, candocevallos@hotmail.com, bryancalisto@yahoo.es

Fecha de recepción: 30 de Junio de 2017 - Fecha de aceptación: 15 de Septiembre de 2017

Resumen: En este estudio experimental se ha determinado la relación existente entre la temperatura del aceite lubricante del motor, el régimen de giro y las aceleraciones mecánicas efectivas RMS (Root Mean Square), al utilizar gasolina tipo extra y una mezcla entre gasolina al 95\% y etanol al 5\% (conocida comercialmente como ecopaís E5); en un vehículo Flex Fuel con motor ciclo Otto, sometiéndolo a una prueba estática y dinámica. Los resultados obtenidos muestran que la temperatura del lubricante tiende a estabilizarse en un mayor tiempo cuando se realiza la prueba con carga al motor, en contraste con el ensayo sin carga. Por otro lado, dicha magnitud incrementa conforme aumenta el régimen de giro para los dos casos; siendo esta menor, de manera general, al utilizar la mezcla combustible a base de gasolina y etanol. Para el caso de las aceleraciones mecánicas, se cumple una tendencia similar, puesto que son menores, habitualmente, al utilizar ecopaís E5.

Palabras clave: etanol; gasolina; temperatura; régimen de giro; aceleraciones mecánicas efectivas

\begin{abstract}
In this experimental investigation, the relationship between engine oil temperature, rotation regime and effective mechanical RMS (Root Mean Square) accelerations was determined with the use of extra gasoline type and a mixture of $95 \%$ gasoline and $5 \%$ ethanol (commercially known as ecopaís E5); in a Flex Fuel vehicle with Otto cycle engine, under static and dynamic test conditions. The obtained results show that the lubricant temperature tends to stabilize in a longer time when the test is carried out with load to the engine, in contrast to the test without load. On the other hand, this magnitude increases as the regime rises for both cases; being lower, with the use of the fuel blend instead of neat gasoline. For the case of mechanical accelerations, it follows a similar trend, since they are, usually, smaller when using ecopaís E5.
\end{abstract}

Key words: ethanol; gasoline; temperature; rotation regime; effective mechanical accelerations 


\section{Introducción}

Cuando se habla respecto al desempeño y eficiencia de un motor de combustión interna, se conoce que el aceite lubricante es considerado como un factor muy influyente en materia de viscosidad, temperatura, espesor, características químicas y otros factores adicionales.

Estudios han demostrado que, de manera general, al tener un aumento en la temperatura del lubricante, su viscosidad tiende a disminuir, ocasionando menor fricción entre componentes móviles pertenecientes a la máquina térmica (Harigaya, Suzuki, \& Takiguchi, 2003; Severa, Havlíček, \& Kumbár, 2014) . La fricción interna del motor puede llegar a representar el 50\% del total de las pérdidas mecánicas (Macián, Tormos, Ruiz, \& Miró, 2016). A su vez, un cambio de temperatura de tan sólo $100{ }^{\circ} \mathrm{C}$, puede causar una variación de hasta 1000 veces en la viscosidad del aceite (Rasid, Mohamad, Ghazali, \& Mahmood, 2012). El régimen del motor es un factor clave que influirá en los cambios de temperatura del lubricante. Se conoce que el régimen de giro es directamente proporcional a la rapidez con la que la temperatura del aceite tienda a variar (Cipollone, Di Battista, \& Mauriello, 2015). A valores mayores de temperatura del lubricante, se consigue incrementos en los valores de torque y potencia del motor; y una reducción en el consumo de combustible (Burnete, Moldovanu, \& Baldean, 2013). Como un dato adicional, el aceite lubricante toma un menor tiempo en calentarse, en contraste con el refrigerante del motor (Cipollone et al., 2015). Es importante mencionar que el nivel de temperatura es uno de los factores más influyentes en la degradación del aceite (Diaby, Sablier, Le Negrate, El Fassi, \& Bocquet, 2009).

El espesor de la película del aceite está relacionado con la viscosidad del mismo, su presión de operación dentro del circuito de lubricación, y el régimen del motor. A mayor viscosidad, el espesor aumentará. En los ciclos de admisión y escape se tendrá mayores espesores en comparación a los ciclos de compresión y expansión (variación de temperatura) (Harigaya et al., 2003). El incremento de la presión de lubricación provocará que la viscosidad sea mayor (Bair, 2000; Bair, Jarzynski, \& Winer, 2001), aumentando de igual manera el espesor. Estudios han demostrado que conforme incrementa las revoluciones de giro, el espesor de la película de aceite tiende a aumentar, y su viscosidad a disminuir, aludiendo este fenómeno persistente a diferentes hipótesis (Harigaya et al., 2003).

Producto del deslizamiento vertical que realiza el pistón, se produce una pequeña capa de aceite adherida a la pared del cilindro. En las fases de admisión y compresión, se produce una emulsión entre esta capa y parte del combustible que ingresa (de Albuquerque, de Andrade Ávila, Barros Zárante, \& Sodré, 2011). Cuando se utiliza combustible con contenido de etanol, se conoce que en una mezcla producida a $100^{\circ} \mathrm{C}$ entre el aceite y este hidrocarburífero, ocasionará una evaporación rápida y total del etanol; a su vez, la contaminación de etanol en el aceite produce la disminución del espesor de la película lubricante; fenómeno denegado cuando se produce la evaporación de este alcohol etílico por la temperatura del aceite (Costa \& Spikes, 2016).

Al referirnos a vibraciones acústicas y mecánicas generadas por el funcionamiento del motor, el impacto del pistón contra la pared del cilindro en sus diferentes modos (fuerzas laterales), juega un papel importante en la vibración horizontal del motor (Albarbar, Gu, Ball, \& 
Starr, 2007). Por otro lado, al disminuir la fricción entre componentes del motor (por disminución de viscosidad en el aceite), se generará un menor ruido (Rasid et al., 2012).

Esta investigación forma parte de un macro estudio, en el cual se han conseguido resultados con relación a emisiones, torque y potencia, vibraciones del motor y temperatura del aceite lubricante; en un vehículo Flex Fuel, al utilizar dos tipos de combustible, sometidos a dos diferentes modalidades de prueba. Sin embargo, en este documento se expone solamente la relación generada entre la temperatura del aceite, el régimen del motor y las aceleraciones mecánicas efectivas $R M S$.

\section{Especificaciones del Vehículo}

\section{Materiales}

En la tabla 1 se exponen las características del vehículo utilizado para el estudio. El aceite lubricante equipado en el motor, de tipo sintético, tiene un índice de viscosidad 20W50.

Tabla 1. Características principales del vehículo utilizado.

\begin{tabular}{ll}
\hline Parámetro & Descripción \\
\hline Modelo & Ford F150 4X2 XLT Flex Fuel \\
Tipo Motor & Ciclo OTTO \\
Cilindraje & $3700 \mathrm{cc}$ \\
Cilindros & V6 \\
Relación de Compresión & $10,5: 1$ \\
$\mathrm{~N}^{\circ}$ Válvulas & 24 \\
Máxima potencia & $302 \mathrm{Hp} @ 6500 \mathrm{RPM}$ \\
Máximo Torque & $278 \mathrm{Lb}-\mathrm{Ft} @$ $4000 \mathrm{RPM}$ \\
Tracción & Posterior \\
Transmisión & 6 velocidades (automático) \\
Tipo aceite & 20W50 (sintético) \\
\hline
\end{tabular}

\section{Especificaciones de los Combustibles}

En la tabla 2 se exponen las características principales de los dos combustibles utilizados en los ensayos: gasolina tipo extra (88,4 octanos RON), y una mezcla entre gasolina y etanol al 5\%, conocida como acopias E5 (89,3 octanos RON) (Gutiérrez, Iñiguez, Cadena, \& Santiana, 2017)

Tabla 2. Características principales de los combustibles

\begin{tabular}{lll}
\hline Combustible & \% de Etanol & Nombre comercial \\
\hline Nafta & $0 \%$ & Gasolina tipo extra \\
Mezcla Nafta + Etanol & $5 \%$ & Ecopaís \\
\hline
\end{tabular}

\section{Especificaciones de Equipos}

Para la obtención de los valores de temperatura del aceite, se utilizó el instrumento de medición de gases Brain Bee AGS-688, el cual brinda la posibilidad de obtener dicha magnitud, mediante una sonda PT100. El instrumento capta temperaturas en el rango de 20 a $150{ }^{\circ} \mathrm{C}$, con una resolución de $1{ }^{\circ} \mathrm{C}$.

Se ha utilizado un dinamómetro de chasís, de 4 rodillos, para la simulación de los métodos de ensayos. El equipo utilizado para la medición de vibraciones del motor responde a las mismas características de una de las investigaciones experimentales que forman parte del 
macro estudio expuesto en un inicio, en el cual se analizan los efectos de las oscilaciones mecánicas generadas por los dos combustibles utilizados (Gutiérrez et al., 2017).

\section{Métodos}

El estudio ha sido dividido en dos etapas: 1) Prueba estática, variando el régimen del motor desde 1000 a 4000 rpm, incluyendo el valor a ralentí; y 2) Prueba dinámica, a las mismas revoluciones. La toma de medidas se ha realizado en intervalos de $1000 \mathrm{rpm}$.

Para las pruebas estática y dinámica, se ha colocado al vehículo sobre el dinamómetro de chasis, con la diferencia de que en la primera prueba indicada lógicamente la transmisión del vehículo no genera movimiento a los rodillos. Para el segundo tipo de prueba, la carga aplicada a la transmisión tan solo fue la resistencia que presentan los rodillos a su libre giro; razón por la cual este valor ha sido despreciado durante el análisis de los resultados. La sonda PT100 fue introducida en el compartimiento de la bayoneta del aceite, respetando su longitud.

\section{Resultados}

En las tablas 3 y 4 se detallan los valores obtenidos referentes a temperatura de aceite y aceleraciones efectivas $R M S$, en relación con el régimen del motor, tanto para la prueba estática como para la dinámica.

Tabla 3. Resultados para la prueba estática

\begin{tabular}{|c|c|c|c|c|}
\hline & \multicolumn{2}{|c|}{ Con GASOLINA } & \multicolumn{2}{|c|}{ Con ECOPAÍS } \\
\hline $\begin{array}{l}\text { Régimen Motor } \\
\text { rpm }\end{array}$ & $\begin{array}{l}\text { Temperatura aceite } \\
{ }^{\circ} \mathrm{C}\end{array}$ & $\begin{array}{c}\text { Aceleraciones RMS } \\
\mathrm{m} / \mathrm{s}^{2}\end{array}$ & $\begin{array}{c}\text { Temperatura aceite } \\
{ }^{\circ} \mathrm{C}\end{array}$ & $\begin{array}{c}\text { Aceleraciones RMS } \\
\mathrm{m} / \mathrm{s}^{2}\end{array}$ \\
\hline Ralentí & 97 & 0,1653 & 99 & 0,2208 \\
\hline 1000 & 98 & 0,2626 & 101 & 0,3152 \\
\hline 2000 & 107 & 2,0352 & 105 & 1,1980 \\
\hline 3000 & 112 & 1,8102 & 110 & 2,3005 \\
\hline 4000 & 120 & 1,7096 & 117 & 1,4170 \\
\hline
\end{tabular}

Tabla 4. Resultados para la prueba dinámica

\begin{tabular}{ccccc}
\hline & \multicolumn{2}{c}{ Con GASOLINA } & \multicolumn{2}{c}{ Con ECOPAÍ́S } \\
\hline Régimen Motor & $\begin{array}{c}\text { Temperatura aceite } \\
\text { rpm }\end{array}{ }^{\circ} \mathrm{C}$ & Aceleraciones RMS & Temperatura aceite & Aceleraciones RMS \\
1000 & 112 & $\mathrm{~m} / \mathrm{s}^{2}$ & ${ }^{\circ} \mathrm{C}$ & $\mathrm{m} / \mathrm{s}^{2}$ \\
2000 & 112 & 0,3831 & 111 & 0,3083 \\
3000 & 113 & 1,9561 & 112 & 1,2484 \\
4000 & 123 & 2,4158 & 117 & 2,1426 \\
\hline
\end{tabular}

\section{Temperatura Aceite}

\section{Análisis de Resultados}

Para la prueba estática realizada, funcionando el motor con combustible gasolina tipo extra y el denominado ecopaís (mezcla gasolina y etanol); a regímenes de velocidad desde ralentí hasta $1000 \mathrm{rpm}$, la temperatura del lubricante fue mayor cuando se utilizó combustible ecopaís, en contraste con el ensayo con gasolina. Una de las posibles causas está relacionada a la mayor fricción entre componentes internos, debido a la baja viscosidad del aceite en estos puntos (Burnete et al., 2013), lo que podría ocasionar que el incremento de calor generado por el trabajo entre dichas partes se transmita hacia el aceite. Para velocidades desde 2000 a $4000 \mathrm{rpm}$, la temperatura fue mayor en el ensayo con gasolina. Con ecopaís, el comportamiento de la 
variación de temperatura del aceite es más estable. De manera general, la temperatura del aceite con ecopaís es menor, debido a que dicho combustible posee menor poder calorífico (Cooney, Yeliana, Worm, \& Naber, 2009). En las figuras 1 y 2 se muestra el análisis en mención.

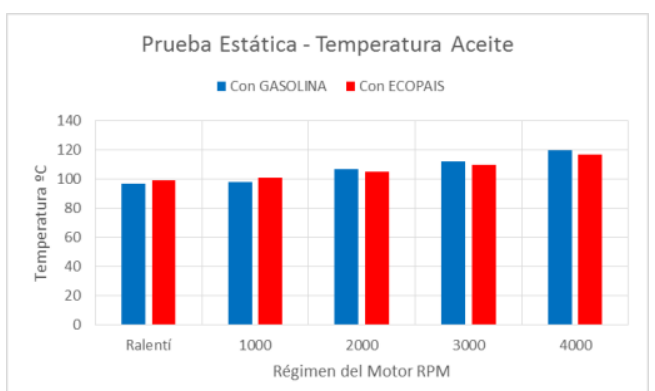

Figura 1. Efectos del combustible ecopaís y gasolina extra, en la temperatura del aceite (prueba estática - gráfico 1).

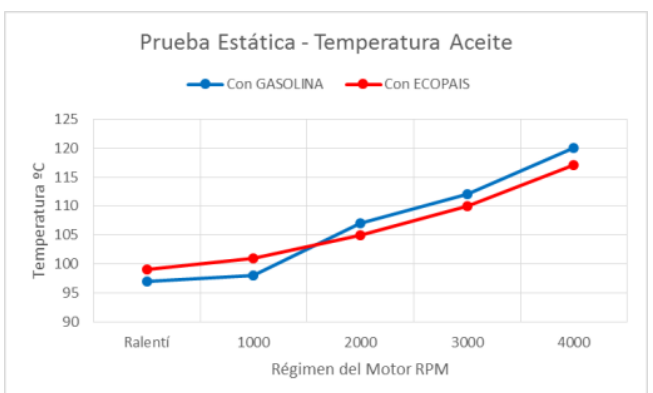

Figura 2. Efectos del combustible ecopaís y gasolina extra, en la temperatura del aceite (prueba estática - gráfico 2).

Para la prueba dinámica, a velocidades desde 1000 a $2000 \mathrm{rpm}$, la temperatura es menor con ecopaís. Desde 2000 hasta valores superiores a $3000 \mathrm{rpm}$, la temperatura es mayor con ecopaís (muestra de una demora en su estabilización). En 4000 rpm, dicha temperatura decrece. Una causa posible de este descenso se debe a que el etanol proporciona a la mezcla un mayor valor de octanaje y mayor velocidad de llama (Cooney et al., 2009), ocasionando disminuir las pérdidas de energía del combustible en calor no aprovechado; y a su vez, reducir la generación de calor producto de la fricción entre el pistón y pared del cilindro, siendo esta mayor cuando se da el golpeteo no controlado. En las figuras 3 y 4 se muestra el análisis en mención.

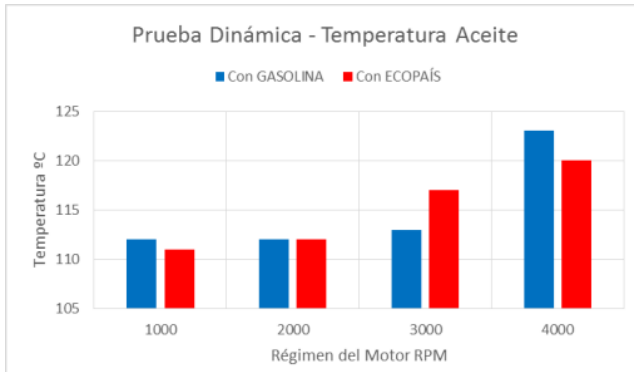

Figura 3. Efectos del combustible ecopaís y gasolina extra, en la temperatura del aceite (prueba dinámica - gráfico $1)$. 


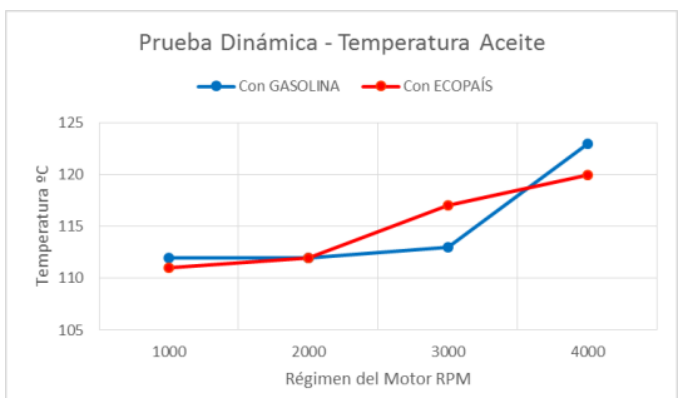

Figura 4. Efectos del combustible ecopaís y gasolina extra, en la temperatura del aceite (prueba dinámica - gráfico

\section{Aceleración RMS (vibraciones)}

Para la prueba estática, en velocidades desde ralentí a $1000 \mathrm{rpm}$, los valores de aceleración $R M S$ son mayores con el uso de ecopaís, pero en mínimas cantidades (despreciables). Desde 1000 a velocidades superiores a $2000 \mathrm{rpm}$, los valores $R M S$ son considerablemente menores en comparación con gasolina (posible causa valor de octanaje (Cooney et al., 2009). En 3000 rpm, el valor es mayor con ecopaís (posiblemente, en este punto se dio el fenómeno de emulsión entre el aceite y el combustible con etanol que, al estar el aceite a una temperatura de $110^{\circ} \mathrm{C}$, la cantidad de alcohol se evaporó, afectando a la calidad de combustión (Costa \& Spikes, 2016), y tiende a decrecer con un régimen hasta $4000 \mathrm{rpm}$. La posible causa de mayor aceleración efectiva $R M S$ con gasolina se debe a que la viscosidad del lubricante disminuye conforme aumenta la temperatura, ocasionando un mayor adelgazamiento de la película de aceite que generará que el contacto entre superficies de partes móviles produzca un incremento en las vibraciones del motor debido a su fricción (Macián et al., 2016; Severa et al., 2014). En las figuras 5 y 6 se muestra el análisis en mención.

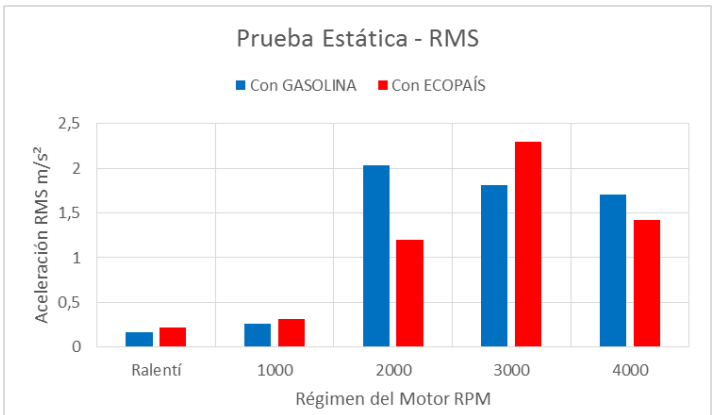

Figura 5. Efectos del combustible ecopaís y gasolina extra, en la aceleración $R M S$ (prueba estática - gráfico 1).

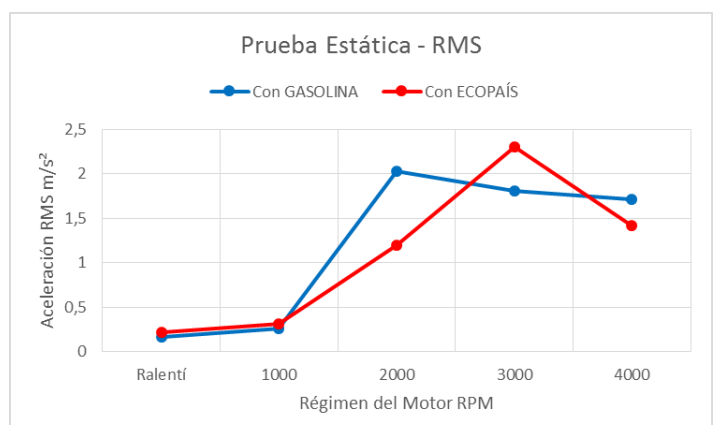

Figura 6. Efectos del combustible ecopaís y gasolina extra, en la aceleración $R M S$ (prueba estática - gráfico 2). 
Para la prueba dinámica, a 1000 rpm el comportamiento de la aceleración para los dos casos es semejante; conforme se aumenta el régimen del motor, se empieza a generar una brecha significativa hasta los $3000 \mathrm{rpm}$. A partir de este punto, para los dos casos, la aceleración decrece casi con la misma tendencia, y en valores similares. Estudios demuestran que conforme aumenta el régimen del motor, a pesar de que la viscosidad del aceite disminuye, el espesor del lubricante aumenta (Harigaya et al., 2003). Una posible razón de la disminución de la aceleración $R M S$ se debe a que a temperaturas y regímenes del motor altos, el caudal de fuga que atraviesa por los taladros de lubricación, supera al que proporcionará la bomba de aceite; lo que ocasiona que el espesor de la película lubricante crezca y disminuya así el espacio entre componentes móviles del motor (Rasid et al., 2012). En las figuras 7 y 8 se muestra el análisis en mención.

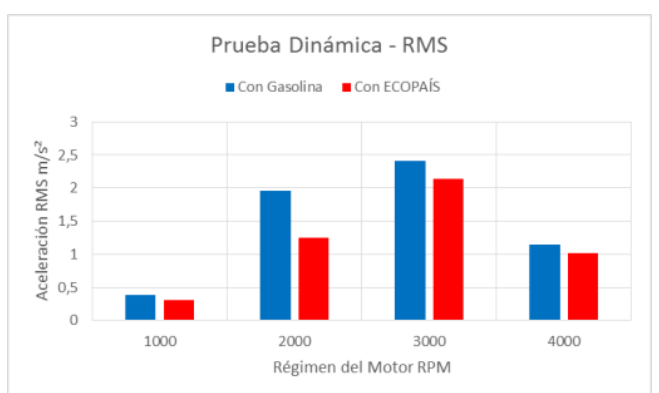

Figura 7. Efectos del combustible ecopaís y gasolina extra, en la aceleración $R M S$ (prueba dinámica - gráfico 1).

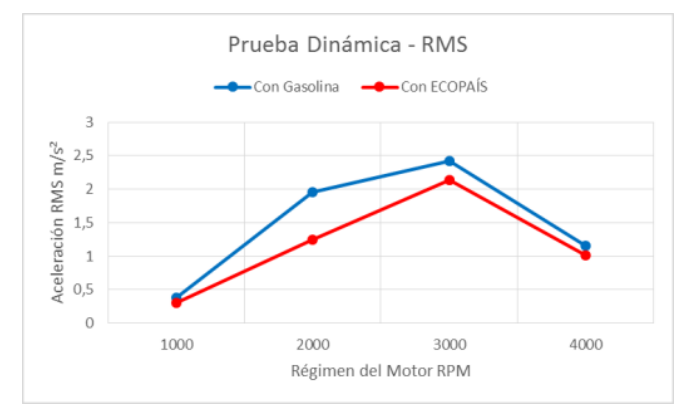

Figura 8. Efectos del combustible ecopaís y gasolina extra, en la aceleración $R M S$ (prueba dinámica - gráfico 2).

\section{Conclusiones}

Respecto a la temperatura de aceite, ésta tiende a estabilizarse en un mayor tiempo cuando se aplica carga al motor, a diferencia del funcionamiento sin carga; sin embargo, la temperatura para los dos casos tiende a llegar al mismo punto.

De manera general, la temperatura del aceite al utilizar ecopaís es menor, con respecto a cuándo se utiliza gasolina. Para los dos casos, la temperatura siempre tenderá a aumentar conforme incremente el régimen de giro del motor. En relación con las aceleraciones mecánicas efectivas $R M S$, éstas son menores cuando se utiliza ecopaís. A velocidades de giro elevadas, para los dos tipos de combustibles, dichas aceleraciones disminuyen.

\section{Bibliografía}

Albarbar, A., Gu, F., Ball, A., \& Starr, A. (2007). Internal combustion engine lubricating oil condition monitoring based on vibro-acoustic measurements. Insight - Non-Destructive Testing and Condition Monitoring, 49(12), 715-718. https://doi.org/10.1784/insi.2007.49.12.715 
Bair, S. (2000). Pressure-Viscosity Behavior of Lubricants to $1.4 \mathrm{GPa}$ and Its Relation to EHD Traction. Tribology Transactions, 43(1), 91-99. https://doi.org/10.1080/10402000008982317

Bair, S., Jarzynski, J., \& Winer, W. O. (2001). The temperature, pressure and time dependence of lubricant viscosity. Tribology International, 34(7), 461-468. https://doi.org/10.1016/S0301679X(01)00042-1

Burnete, N., Moldovanu, D., \& Baldean, D. (2013). Studies and Researches Regarding the Influence of Lubricating Oil Temperature on Diesel Engines. Acta Technica Corviniensis-Bulletin of Engineering, 6(3), 55-57.

Cipollone, R., Di Battista, D., \& Mauriello, M. (2015). Effects of Oil Warm up Acceleration on the Fuel Consumption of Reciprocating Internal Combustion Engines. Energy Procedia, 82, 1-8. https://doi.org/10.1016/j.egypro.2015.11.870

Cooney, C. P., Yeliana, Worm, J. J., \& Naber, J. D. (2009). Combustion Characterization in an Internal Combustion Engine with Ethanol-Gasoline Blended Fuels Varying Compression Ratios and Ignition Timing. Energy \& Fuels, 23(5), 2319-2324. https://doi.org/10.1021/ef800899r

Costa, H. L., \& Spikes, H. A. (2016). Impact of ethanol on the formation of antiwear tribofilms from engine lubricants. Tribology International, 93, 364-376. https://doi.org/10.1016/j.triboint.2015.09.021

de Albuquerque, P. C. C., de Andrade Ávila, R. N., Barros Zárante, P. H., \& Sodré, J. R. (2011). Lubricating oil influence on exhaust hydrocarbon emissions from a gasoline fueled engine. Tribology International, 44(12), 1796-1799. https://doi.org/10.1016/j.triboint.2011.07.003

Diaby, M., Sablier, M., Le Negrate, A., El Fassi, M., \& Bocquet, J. (2009). Understanding carbonaceous deposit formation resulting from engine oil degradation. Carbon, 47(2), 355-366. https://doi.org/10.1016/j.carbon.2008.10.014

Gutiérrez, M., Iñiguez, J., Cadena, X., \& Santiana, G. (2017). Análisis de las Vibraciones de un Motor Ciclo Otto con una Mezcla Combustible a Base de Gasolina y de Etanol. INNOVA Research Journal.

Harigaya, Y., Suzuki, M., \& Takiguchi, M. (2003). Analysis of Oil Film Thickness on a Piston Ring of Diesel Engine: Effect of Oil Film Temperature. Journal of Engineering for Gas Turbines and Power, 125(2), 596-603. https://doi.org/10.1115/1.1501078

Macián, V., Tormos, B., Ruiz, S., \& Miró, G. (2016). Low viscosity engine oils: Study of wear effects and oil key parameters in a heavy duty engine fleet test. Tribology International, 94, 240-248. https://doi.org/10.1016/j.triboint.2015.08.028

Rasid, A. F. A., Mohamad, T. I., Ghazali, M. J., \& Mahmood, W. M. F. W. (2012). Effects of lubricant temperature in a motorized engine. World Applied Sciences Journal, 20(7), 927-930. https://doi.org/10.5829/idosi.wasj.2012.20.07.2737

Severa, L., Havlíček, M., \& Kumbár, V. (2014). Temperature dependent kinematic viscosity of different types of engine oils. Acta Universitatis Agriculturae et Silviculturae Mendelianae Brunensis, 57(4), 95-102. https://doi.org/10.11118/actaun200957040095 